\title{
PENENTUAN SUATU GRUP KUOSIEN FUZZY DARI SUATU GRUP
}

\author{
PUTRI ELIZA, NOVA NOLIZA BAKAR \\ Program Studi Matematika, \\ Fakultas Matematika dan Ilmu Pengetahuan Alam, Universitas Andalas, \\ Kampus UNAND Limau Manis Padang, Indonesia, \\ email : putrielizha56@gmail.com
}

\begin{abstract}
Abstrak. Pada tulisan ini akan dibahas tentang bagaimana menentukan suatu grup kuosien fuzzy dari suatu grup. Untuk itu, diperlukan konsep-konsep tentang grup, subgrup normal, koset, himpunan fuzzy, subgrup normal fuzzy dan koset fuzzy. Diberikan $G$ adalah grup, pemetaan $\mu: G \longrightarrow[0,1]$ disebut himpunan fuzzy dari $G$. Diberikan $K, N$ subgrup normal dari $G$ dan $\zeta$ adalah himpunan semua koset fuzzy dari $G$, maka pemetaan $\hat{\mu}: G / K \longrightarrow[0,1]$ dan $\bar{\mu}: \zeta \longrightarrow[0,1]$ merupakan suatu grup kuosien fuzzy.

Kata Kunci: Grup kuosien fuzzy, grup, subgrup normal, koset, himpunan fuzzy, subgrup normal fuzzy, koset fuzzy
\end{abstract}

\section{Pendahuluan}

Grup kuosien merupakan salah satu materi dalam mata kuliah struktur aljabar yang meliputi konsep grup, subgrup, subgrup normal, dan koset. Misalkan $G$ bukan himpunan kosong dan $G$ adalah suatu grup, dan misalkan $N$ adalah subgrup normal dari grup $G$, maka $G / N=\{N g \mid g \in G\}$ juga suatu grup. Dalam hal ini, $G / N$ disebut grup kuosien [3].

Pada tahun 1965, Prof. L.A. Zadeh pertama kali memperkenalkan tentang himpunan fuzzy. Zadeh mendefinisikan suatu himpunan fuzzy $\mu$ dalam $X$ dengan fungsi keanggotaan $f_{\mu}(x)$, dimana nilai keanggotaan dari elemen-elemennya adalah bilangan riil dalam interval $[0,1]$ [7]. Teori tentang himpunan fuzzy juga dikembangkan pada struktur aljabar. Studi tentang struktur aljabar fuzzy pertama kali diperkenalkan oleh Rosenfield pada tahun 1972 [2]. Subgrup fuzzy dan subgrup normal fuzzy merupakan salah satu studi yang dipelajari dalam struktur aljabar fuzzy.

Pada tahun 1995, Hee Chan Choi dalam papernya membahas tentang grup kuosien fuzzy. Grup, subgrup normal, subgrup fuzzy, subgrup normal fuzzy, dan koset merupakan teori-teori yang digunakan dalam pembahasannya. Konsep dari grup kuosien fuzzy berbeda dengan konsep grup kuosien. Untuk itu, pada penulisan ini akan dijelaskan bagaimaana menentukan suatu grup kuosien fuzzy dari suatu grup.

\section{Himpunan Fuzzy}

Definisi 2.1. [7] Misalkan X suatu himpunan yang tak kosong. Himpunan fuzzy $\mu$ atas $X$ didefinisikan sebagai: 


$$
\mu=\left\{\left\langle x, f_{\mu}(x)\right\rangle: x \in X\right\}
$$

dimana $f_{\mu}: X \longrightarrow[0,1]$, dan $f_{\mu}(x)$ disebut derajat keanggotaan dari $x$ pada himpunan fuzzy $\mu$.

Definisi 2.2. [2] Misalkan $G$ suatu himpunan tak kosong. Fungsi $\mu: G \rightarrow[0,1]$ disebut suatu himpunan fuzzy dari $G$.

\section{Subgrup Fuzzy dan Subgrup Normal Fuzzy}

Definisi 3.1. [2] Himpunan fuzzy $\mu$ dari grup $G$ disebut subgrup fuzzy jika:

(i) $\mu(x y) \geqslant \min \{\mu(x), \mu(y)\}$ untuk setiap $x, y \in G$.

(ii) $\mu\left(x^{-1}\right)=\mu(x)$ untuk setiap $x \in G$,

dengan $\mu(e) \geqslant \mu(x)$ untuk setiap $x \in G$, dimana e adalah elemen identitas dari $G$.

Definisi 3.2. [5] Misalkan $G$ grup, subgrup fuzzy $\mu$ dari $G$ disebut subgrup normal fuzzy jika $\mu(x)=\mu\left(y^{-1} x y\right)$, untuk setiap $x, y \in G$.

\section{Grup Kuosien Fuzzy}

Teorema 4.1. [2] Misalkan $\mu$ adalah subgrup normal fuzzy dari grup $G$ dengan identitas e. Misalkan $K=\{x \in G \mid \mu(x)=\mu(e)\}$ maka $K$ subgrup normal dari $G$. Pandang suatu pemetaan $\hat{\mu}: G / K \longrightarrow[0,1]$ yang didefinisikan oleh

$$
\hat{\mu}(K x)=\sup _{k \in K} \mu(k x), \forall x \in G .
$$

Maka $\hat{\mu}$ terdefinisi dengan baik dan $\hat{\mu}$ adalah subgrup fuzzy dari $G / K$, dimana $G / K=\{K x \mid x \in G\}$

Bukti. Misalkan $\mu$ adalah subgrup normal fuzzy dari grup $G$ dengan identitas $e$.

(1) Misalkan $K=\{x \in G \mid \mu(x)=\mu(e)\}$. Akan ditunjukkan bahwa $K$ subgrup normal dari $G$. Berdasarkan Definisi 3.2, karena $\mu$ subgrup normal fuzzy maka $\mu\left(y^{-1} x y\right)=\mu(x)=\mu\left(y x y^{-1}\right)=\mu(e)$ untuk setiap $x \in K$ dan $y \in G$. Karena $y x y^{-1} \in G$ dan $\mu\left(y x y^{-1}\right)=\mu(e)$ maka $y x y^{-1} \in K$, sehingga dapat disimpulkan bahwa $K$ adalah subgrup normal dari $\mathrm{G}$.

(2) Selanjutnya, akan ditunjukkan bahwa $\hat{\mu}$ terdefinisi dengan baik.

Ambil $x, y \in G / K$ dengan $x=y$. Akan ditunjukkan bahwa $\hat{\mu}(x)=\hat{\mu}(y)$.

Dari $x \in G / K$ diperoleh $x=K a$ untuk suatu $a \in G$, dan dari $y \in G / K$ diperoleh $y=K b$ untuk suatu $b \in G$, sehingga $K a=K b$.

Misal $z \in K a$ dan $z \in K b$ maka

$$
\begin{aligned}
& z=k_{1} a \text { untuk suatu } k_{1} \in K, \\
& z=k_{2} b \text { untuk suatu } k_{2} \in K .
\end{aligned}
$$

Jadi, $k_{1} a=k_{2} b$ sehingga diperoleh $a=k_{1}^{-1} k_{2} b=k_{3} b$ untuk suatu $k_{3}=k_{1}^{-1} k_{2} \in$ $K$. 
Perhatikan bahwa:

$$
\begin{aligned}
\hat{\mu}(x) & =\hat{\mu}(K a), \\
& =\sup _{k \in K} \mu(k a), \\
& =\sup _{k \in K} \mu\left(k\left(k_{3} b\right)\right), \\
& \left.=\sup _{k \in K} \mu\left(\left(k k_{3}\right) b\right)\right), \\
& =\sup _{k_{4} \in K} \mu\left(k_{4} b\right), \text { untuk suatu } k_{4}=k k_{3} \in K, \\
& =\hat{\mu}(K b), \\
& =\hat{\mu}(y) .
\end{aligned}
$$

Terbukti bahwa $\hat{\mu}$ terdefinisi dengan baik.

(3) Selanjutnya, akan dibuktikan bahwa $\hat{\mu}$ subgrup fuzzy dari $G / K$. Subgrup $\hat{\mu}$ dikatakan subgrup fuzzy dari $G / K$ jika untuk setiap $K x, K y \in G / K$ berlaku:

(a) $\hat{\mu}(K x K y) \geqslant \min \{\hat{\mu}(K x), \hat{\mu}(K y)\}$.

(b) $\hat{\mu}\left((K x)^{-1}\right)=\hat{\mu}(K x)$,

dimana $(K x)^{-1}$ adalah simbol untuk invers dari $K x$.

Perhatikan bahwa:

$$
\begin{aligned}
\hat{\mu}(K x K y) & =\hat{\mu}(K x y), \text { karena } K \text { subgrup normal dari } G, \\
& =\sup _{k \in K} \mu(k x y), \\
& \geqslant \sup _{k_{1}, k_{2} \in K} \min \left\{\mu\left(k_{1} x\right), \mu\left(k_{2} y\right)\right\} \\
& \geqslant \min \left\{\sup _{k_{1} \in K} \mu\left(k_{1} x\right), \sup _{k_{2} \in K} \mu\left(k_{2} y\right)\right\}, \\
& =\min \{\hat{\mu}(K x), \hat{\mu}(K y)\}, \\
\hat{\mu}\left((K x)^{-1}\right) & =\hat{\mu}\left(K x^{-1}\right), \\
& =\sup _{k \in K} \mu\left(k x^{-1}\right), \\
& =\sup _{k \in K} \mu(k x), \\
& =\hat{\mu}(K x) .
\end{aligned}
$$

Berdasarkan (4.1) dan (4.2) terbukti bahwa $\hat{\mu}$ subgrup fuzzy dari $G / K$.

Definisi 4.2. [2] Misalkan $\mu$ adalah subgrup normal fuzzy dari grup $G$ dengan identitas e. Fungsi $\hat{\mu}: G / K \longrightarrow[0,1]$ didefinisikan oleh

$$
\hat{\mu}(K x)=\sup _{k \in K} \mu(k x), \forall x \in G,
$$

dengan $K=\{x \in G \mid \mu(x)=\mu(e)\}$ adalah subgrup normal dari $G$. Dalam hal ini, $\hat{\mu}$ disebut grup kuosien fuzzy dari $\mu$ oleh $K$.

Definisi 4.3. [2] Misalkan $\mu$ adalah subgrup fuzzy dari grup $G$. Untuk setiap $x \in G$, didefinisikan pemetaan $\hat{\mu}_{x}: G \rightarrow[0,1]$ oleh

$$
\hat{\mu}_{x}(g)=\mu\left(g x^{-1}\right), \forall g \in G \text {. }
$$


Dalam hal ini, $\hat{\mu}_{x}$ disebut koset fuzzy dari $G$.

Teorema 4.4. [2] Misalkan $N$ subgrup normal dari grup $G$ dan $\mu$ adalah subgrup normal fuzzy dari $G$. Misal $\zeta$ adalah himpunan semua koset fuzzy dari $G$ oleh $\mu$. Maka $\zeta=\left\{\hat{\mu}_{x} \mid x \in G, \hat{\mu}_{x}\right.$ adalah koset fuzzy dari $\left.G\right\}$ adalah suatu grup terhadap operasi komposisi yang didefinisikan oleh

$$
\hat{\mu}_{x} \circ \hat{\mu}_{y}=\hat{\mu}_{x y}, \forall x, y \in G .
$$

Definisikan fungsi $\bar{\mu}: \zeta \rightarrow[0,1]$ sebagai:

$$
\bar{\mu}\left(\hat{\mu}_{x}\right)=\sup _{n \in N} \hat{\mu}_{x}(n)=\sup _{n \in N} \mu\left(n x^{-1}\right), \forall x \in G .
$$

Maka $\bar{\mu}$ adalah subgrup fuzzy dari $\zeta$. Dalam hal ini, $\bar{\mu}$ adalah grup kuosien fuzzy dari $\mu$ oleh $N$.

Bukti. Misalkan $N$ subgrup normal dari grup $G$ dan $\mu$ adalah subgrup normal fuzzy dari $G$.

(1) Misalkan $\zeta=\left\{\hat{\mu}_{x} \mid x \in G, \hat{\mu}_{x}\right.$ adalah koset fuzzy dari $\left.G\right\}$ adalah suatu grup terhadap operasi komposisi yang didefinisikan oleh persamaan (4.3). Akan ditunjukkan bahwa persamaan (4.3) terdefinisi dengan baik.

Ambil $x, y, x_{0}, y_{0} \in G$ sedemikian sehingga

$$
\hat{\mu}_{x}=\hat{\mu}_{x_{0}}, \hat{\mu}_{y}=\hat{\mu}_{y_{0}} .
$$

Maka akan ditunjukkan bahwa

$$
\hat{\mu}_{x} \circ \hat{\mu}_{y}=\hat{\mu}_{x_{0}} \circ \hat{\mu}_{y_{0}}
$$

yaitu, $\hat{\mu}_{x y}=\hat{\mu}_{x_{0} y_{0}}$.

Berdasarkan Definisi 3.2, dapat diperoleh:

$$
\begin{aligned}
\hat{\mu}_{x y}(g) & =\mu\left(g y^{-1} x^{-1}\right), \forall g \in G, \\
\hat{\mu}_{x_{0} y_{0}}(g) & =\mu\left(g y_{0}^{-1} x_{0}^{-1}\right), \quad \forall g \in G .
\end{aligned}
$$

Perhatikan bahwa:

$$
\begin{aligned}
\mu\left(g y^{-1} x^{-1}\right) & =\mu\left(g y_{0}^{-1} y_{0} y^{-1} x^{-1}\right), \\
& =\mu\left(g y_{0}^{-1} x_{0}^{-1} x_{0} y_{0} y^{-1} x^{-1}\right), \\
& =\mu\left(\left(g y_{0}^{-1} x_{0}^{-1}\right)\left(x_{0} y_{0} y^{-1} x^{-1}\right)\right), \\
& \geq \min \left\{\mu\left(g y_{0}^{-1} x_{0}^{-1}\right), \mu\left(x_{0} y_{0} y^{-1} x^{-1}\right)\right\} .
\end{aligned}
$$

Selanjutnya, dari persamaan (4.5) dapat diperoleh:

$$
\begin{aligned}
& \hat{\mu}_{x}(g)=\hat{\mu}_{x_{0}}(g) \text { sehingga } \mu\left(g x^{-1}\right)=\mu\left(g x_{0}^{-1}\right), \forall g \in G, \\
& \hat{\mu}_{y}(g)=\hat{\mu}_{y_{0}}(g) \text { sehingga } \mu\left(g y^{-1}\right)=\mu\left(g y_{0}^{-1}\right), \forall g \in G .
\end{aligned}
$$


Selanjutnya, substitusikan $x_{0} y_{0} y^{-1}$ ke $g$ dalam persamaan (4.8) sehingga diperoleh:

$$
\begin{aligned}
\mu\left(x_{0} y_{0} y^{-1} x^{-1}\right) & =\mu\left(x_{0} y_{0} y^{-1} x_{0}^{-1}\right) \\
& =\mu\left(x_{0}\left(y_{0} y^{-1}\right) x_{0}^{-1}\right) \\
& =\mu\left(y_{0} y^{-1}\right)(\mu \text { subgrup normal fuzzy }) \\
& =\mu\left(y_{0} y_{0}^{-1}\right) \\
& =\mu(e) .
\end{aligned}
$$

Berdasarkan Definisi 3.1, $\mu(e) \geq \mu\left(g y_{0}^{-1} x_{0}^{-1}\right)$, sehingga dari persamaan (4.7) diperoleh:

$$
\begin{aligned}
\mu\left(g y^{-1} x^{-1}\right) & \geq \min \left\{\mu\left(g y_{0}^{-1} x_{0}^{-1}\right), \mu\left(x_{0} y_{0} y^{-1} x^{-1}\right)\right\} \\
& =\min \left\{\mu\left(g y_{0}^{-1} x_{0}^{-1}\right), \mu(e)\right\} \\
& =\mu\left(g y_{0}^{-1} x_{0}^{-1}\right) .
\end{aligned}
$$

Dengan cara yang sama, substitusikan $x y y_{0}^{-1}$ ke $g$ dalam persamaan (4.8). Karena $\mu$ subgrup normal fuzzy, maka

$$
\begin{aligned}
\mu\left(x y y_{0}^{-1} x_{0}^{-1}\right) & =\mu\left(x y y_{0}^{-1} x^{-1}\right) \\
& =\mu\left(x\left(y y_{0}^{-1}\right) x^{-1}\right), \\
& =\mu\left(y y_{0}^{-1}\right) \\
& =\mu(e) .
\end{aligned}
$$

Perhatikan bahwa:

$$
\begin{aligned}
\mu\left(g y_{0}^{-1} x_{0}^{-1}\right) & =\mu\left(g y^{-1} y y_{0}^{-1} x_{0}^{-1}\right) \\
& =\mu\left(g y^{-1} x^{-1} x y y_{0}^{-1} x_{0}^{-1}\right) \\
& =\mu\left(\left(g y^{-1} x^{-1}\right)\left(x y y_{0}^{-1} x_{0}^{-1}\right)\right), \\
& \geq \min \left\{\mu\left(g y^{-1} x^{-1}\right), \mu\left(x y y_{0}^{-1} x_{0}^{-1}\right)\right\} \\
& =\mu\left(g y^{-1} x^{-1}\right) .
\end{aligned}
$$

Dari (4.10) dan (4.11) diperoleh $\mu\left(g y_{0}^{-1} x_{0}^{-1}\right)=\mu\left(g y^{-1} x^{-1}\right)$ yang menunjukkan bahwa $\hat{\mu}_{x y}=\hat{\mu}_{x_{0} y_{0}}$, sehingga terbukti bahwa persamaan (4.3) terdefinisi dengan baik.

(2) Selanjutnya akan dibuktikan bahwa

$$
\zeta=\left\{\hat{\mu}_{x} \mid x \in G, \hat{\mu}_{x} \text { adalah koset fuzzy dari } G\right\}
$$

adalah grup. $\zeta$ dikatakan grup jika:

(a) Untuk setiap $\hat{\mu}_{x_{1}}, \hat{\mu}_{x_{2}} \in \zeta$ maka $\hat{\mu}_{x_{1}} \circ \hat{\mu}_{x_{2}} \in \zeta$.

(b) Jika $\hat{\mu}_{x_{1}}, \hat{\mu}_{x_{2}}, \hat{\mu}_{x_{3}} \in \zeta$ maka $\hat{\mu}_{x_{1}} \circ\left(\hat{\mu}_{x_{2}} \circ \hat{\mu}_{x_{3}}\right)=\left(\hat{\mu}_{x_{1}} \circ \hat{\mu}_{x_{2}}\right) \circ \hat{\mu}_{x_{3}}$.

(c) Terdapat $\hat{\mu}_{e} \in \zeta$ sehingga $\hat{\mu}_{e} \circ \hat{\mu}_{x}=\hat{\mu}_{x} \circ \hat{\mu}_{e}=\hat{\mu}_{x}$, untuk setiap $\hat{\mu}_{x} \in \zeta$.

(d) Untuk setiap $\hat{\mu}_{x} \in \zeta$, terdapat suatu $\left(\hat{\mu}_{x}\right)^{-1} \in \zeta$ sehingga

$$
\left(\hat{\mu}_{x}\right)^{-1} \circ \hat{\mu}_{x}=\hat{\mu}_{x} \circ\left(\hat{\mu}_{x}\right)^{-1}=\hat{\mu}_{e} .
$$

Perhatikan bahwa: 
(a) Ambil $\hat{\mu}_{x_{1}}, \hat{\mu}_{x_{2}} \in \zeta$. Akan ditunjukkan bahwa $\hat{\mu}_{x_{1}} \circ \hat{\mu}_{x_{2}} \in \zeta$.

Perhatikan bahwa:

$$
\begin{aligned}
\hat{\mu}_{x_{1}} \circ \hat{\mu}_{x_{2}}= & \hat{\mu}_{x_{1} x_{2}} \\
= & \hat{\mu}_{x_{3}}, \text { untuk suatu } x_{3}=x_{1} x_{2} \in G . \\
& \hat{\mu}_{x_{1}} \circ \hat{\mu}_{x_{2}} \in \zeta .
\end{aligned}
$$

(b) Ambil $\hat{\mu}_{x_{1}}, \hat{\mu}_{x_{2}}, \hat{\mu}_{x_{3}} \in \zeta$. Akan ditunjukkan

$$
\hat{\mu}_{x_{1}} \circ\left(\hat{\mu}_{x_{2}} \circ \hat{\mu}_{x_{3}}\right)=\left(\hat{\mu}_{x_{1}} \circ \hat{\mu}_{x_{2}}\right) \circ \hat{\mu}_{x_{3}} .
$$

Perhatikan bahwa:

$$
\begin{aligned}
\hat{\mu}_{x_{1}} \circ\left(\hat{\mu}_{x_{2}} \circ \hat{\mu}_{x_{3}}\right) & =\hat{\mu}_{x_{1}} \circ \hat{\mu}_{x_{2} x_{3}} \\
& =\hat{\mu}_{x_{1} x_{2} x_{3}} \\
& =\hat{\mu}_{x_{1} x_{2}} \circ \hat{\mu}_{x_{3}} \\
& =\left(\hat{\mu}_{x_{1}} \circ \hat{\mu}_{x_{2}}\right) \circ \hat{\mu}_{x_{3}} .
\end{aligned}
$$

(c) Karena $G$ suatu grup maka terdapat $e \in G$ sehingga $\hat{\mu}_{e} \in \zeta$. Akan ditunjukkan $\hat{\mu}_{e}$ adalah identitas di $\zeta$.

Perhatikan bahwa:

$$
\begin{aligned}
\hat{\mu}_{e} \circ \hat{\mu}_{x} & =\hat{\mu}_{e x} \\
& =\hat{\mu}_{x}, \text { untuk setiap } \hat{\mu}_{x} \in \zeta, \text { dan } \\
\hat{\mu}_{x} \circ \hat{\mu}_{e} & =\hat{\mu}_{x e} \\
& =\hat{\mu}_{x}, \text { untuk setiap } \hat{\mu}_{x} \in \zeta .
\end{aligned}
$$

Jadi, terdapat $\hat{\mu}_{e} \in \zeta$ sehingga $\hat{\mu}_{e} \circ \hat{\mu}_{x}=\hat{\mu}_{x} \circ \hat{\mu}_{e}=\hat{\mu}_{x}$, untuk setiap $\hat{\mu}_{x} \in \zeta$.

(d) Karena $G$ suatu grup maka untuk setiap $x \in G$ terdapat $x^{-1} \in G$.

Pilih $\left(\hat{\mu}_{x}\right)^{-1}=\hat{\mu}_{x^{-1}}$. Akan ditunjukkan $\hat{\mu}_{x^{-1}}$ adalah invers dari $\hat{\mu}_{x}$.

Perhatikan bahwa:

$$
\begin{aligned}
\hat{\mu}_{x^{-1}} \circ \hat{\mu}_{x} & =\hat{\mu}_{x^{-1} x}, \\
& =\hat{\mu}_{e}, \text { untuk setiap } \hat{\mu}_{x} \in \zeta, \text { dan } \\
\hat{\mu}_{x} \circ \hat{\mu}_{x^{-1}} & =\hat{\mu}_{x x^{-1}}, \\
& =\hat{\mu}_{e}, \text { untuk setiap } \hat{\mu}_{x} \in \zeta .
\end{aligned}
$$

Jadi, untuk setiap $\hat{\mu}_{x} \in \zeta$, terdapat suatu $\hat{\mu}_{x}^{-1} \in \zeta$ sehingga

$$
\hat{\mu}_{x}^{-1} \circ \hat{\mu}_{x}=\hat{\mu}_{x} \circ \hat{\mu}_{x}^{-1}=\hat{\mu}_{e} .
$$

(3) Selanjutnya, akan dibuktikan bahwa $\bar{\mu}$ suatu subgrup fuzzy dari $\zeta$. Misalkan 
$x, y \in G$ maka:

$$
\begin{aligned}
\bar{\mu}\left(\hat{\mu}_{x} \circ \hat{\mu}_{y}\right) & =\bar{\mu}\left(\hat{\mu}_{x y}\right) \\
& =\sup _{n \in N} \mu\left(n y^{-1} x^{-1}\right), \\
& =\sup _{n \in N} \mu\left(n_{1} y^{-1} n_{2} x^{-1}\right), \\
& \geq \sup _{n_{1}, n_{2} \in N} \min \left\{\mu\left(n_{1} y^{-1}\right), \mu\left(n_{2} x^{-1}\right)\right\}, \\
& \geq \min \left\{\sup _{n_{1} \in N} \mu\left(n_{1} y^{-1}\right), \sup _{n_{2} \in N} \mu\left(n_{2} x^{-1}\right)\right\}, \\
& =\min \left\{\sup _{n_{1} \in N} \hat{\mu}_{y}\left(n_{1}\right), \sup _{n_{2} \in N} \hat{\mu}_{x}\left(n_{2}\right)\right\}, \\
& =\min _{\bar{\mu}}\left\{\bar{\mu}\left(\hat{\mu}_{y}\right), \bar{\mu}\left(\hat{\mu}_{x}\right)\right\}, \operatorname{dan} \\
\bar{\mu}\left(\hat{\mu}_{x}^{-1}\right) & =\bar{\mu}\left(\hat{\mu}_{x-1}\right), \\
& =\sup _{n \in N} \hat{\mu}_{x}^{-1}(n), \\
& =\sup _{n \in N} \mu(n x), \\
& =\sup _{n \in N} \mu\left(n x^{-1}\right), \\
& =\sup _{n \in N} \hat{\mu}_{x}(n), \\
& =\bar{\mu}\left(\hat{\mu}_{x}\right) .
\end{aligned}
$$

Dari (4.12 dan (4.13) dapat diperoleh bahwa $\bar{\mu}$ adalah subgrup fuzzy dari $\zeta$, sehingga dapat disimpulkan bahwa $\bar{\mu}$ adalah grup kuosien fuzzy dari $\mu$ oleh $N$.

\section{Ucapan Terima kasih}

Penulis mengucapkan terima kasih kepada Bapak Dr. Admi Nazra, ibu dr. Yanita, Ibu Dr. Susila Bahri, dan Ibu Monika Rianti Helmi, M.Si yang telah memberikan masukan dan saran sehingga paper ini dapat diselesaikan dengan baik.

\section{Daftar Pustaka}

[1] Bartle, R.G dan Donald, R.S. 2000. Introduction to Real Analysis, $3^{\text {th }}$ ed. John Wiley dan Sons, USA.

[2] Choi, H.C. 1990. On Some Fuzzy Quotient Groups. Kangweon-Kyungki Math. Jour. 3(1) : $49-57$.

[3] Herstein, I.N. 1975. Topics in Algebra. $2^{\text {nd }}$ ed. John Wiley dan Sons, New York.

[4] Hila, K. 2008. On Fuzzy Relations and Fuzzy Quotient $\Gamma$-Groups. Quasigroups and Related Systems 16 : $199-206$.

[5] Kandasamy, W.B.V. 2003. Smarandache Fuzzy Algebra. American Research Press Rehoboth, USA.

[6] Ajmal, N. dan Prajapai, A.S. 1992. Fuzzy Cosets and Fuzzy Normal Subgroups. Inform. Sci. $64: 17-25$.

[7] Zadeh, L.A. 1965. Fuzzy Sets. Information and Control $8: 338-353$. 